\title{
La fécondation in vitro et le transfert d'embryon dans l'espèce humaine
}

\author{
D Royère, S Hamamah, ML Couet, J Lansac
}

\begin{abstract}
CHU Bretonneau, dépt de gyn obs et reproduction humaine, unité de biologie de la reproduction, 37044 Tours, France
\end{abstract}

(Reçu le 14 juin 1991, accepté le 20 août 1991)

\begin{abstract}
Résumé - Depuis son apparition au début des années 1980, la fécondation in vitro et le transfert d'embryon chez l'homme ont connu un développement important comme en témoigne l'augmentation du nombre des ponctions réalisées, puisqu'en France, l'enquête nationale fait état entre 1986 et 1989 d'un doublement du nombre des ponctions $(11000 / 24000)$. L'évolution générale de cette technique est marquée par un recul des indications tubaires, passées de 70 à $50 \%$ de l'ensemble des indications. Les étapes particulières qui ont marqué l'évolution de la Fivete concernent les méthodes de stimulation et de ponction, la congélation embryonnaire. Le recueil ovocytaire ne se fait pratiquement plus par cœlioscopie sous anesthésie générale, mais il est effectué par écho-guidage avec l'aide d'une sonde vaginale, sous simple analgésie, lors d'une hospitalisation de jour $(95 \%$ des ponctions). La deuxième étape concerne le recours aux agonistes de la GnRH associés aux hormones gonadotropes. L'objectif attendu de cette méthode était la suppression du pic ovulatoire spontané de LH survenant de façon inappropriée dans 10-15\% des cycles de stimulation. Les résultats moins attendus sont l'augmentation du nombre des ovocytes recueillis et des embryons obtenus, ainsi que l'augmentation nette du taux de grossesse clinique par ponction. Actuellement ce type de traitement est utilisé pour $89 \%$ des ponctions avec un taux de grossesse clinique par ponction de $18,1 \%$. L'obtention d'un nombre plus élevé d'embryons a eu pour conséquences non seulement l'augmentation du nombre d'embryons transférés (2,2-2,9 entre 1986 et 1989), mais aussi le recours accru à la congélation des embryons sumuméraires avec transfert différé, avec un taux de grossesse clinique de $7,5 \%$ par transfert. Parmi les objectifs à court et à moyen terme, domine la diminution du risque de grossesse multiple, lesquelles représentent $23,5 \%$ de l'ensemble des accouchements (20\% gémellaires, 3,5\% triples). Leurs complications (retard de croissance intra-utérin, prématurité, mortalité infantile périnatale) imposent une stratégie de plus en plus rigoureuse de transfert embryonnaire prenant en compte l'âge, l'indication et le déroulement global de la tentative tout en préservant l'efficacité globale de cette technique de procréation médicalement assistée.
\end{abstract}

\section{fécondation in vitro / transfert d'embryon / homme}

Summary - In vitro fertilization and embryo transfer in man. Since the early eighties, in vitro fertilization and embryo transfer in man has been increasingly developed. This development has concerned the number of oocyte retrievals, which increased more than 2-fold between 1986 and 1989 (French register, 11 000/24 000). Another important fact has been the frequency of female tubal factor in in vitro fertilization (IVF) attempts, which has decreased from 70 to $50 \%$ of all indica- 
tions during the last $4 \mathrm{yr}$. Certain steps have markedly modified the IVF procedure, particularly in regard to oocyte recovery, superovulation protocols and embryo freezing. Oocyte retrieval hardly ever requires laparoscopy under general anesthesia, but is more often performed using echoguidance via the vaginal route, under simple analgesia with only $1 d$ of hospital care (95\% of all retrievals). A second step concerned the combined use of GnRH agonists with gonadotropins. The aim of such treatment was to prevent the early spontaneous gonadotropin surge which accounted for $10-15 \%$ of treatment cycles. Surprisingly, an increase both in the number of collected oocytes and the number of embryos was obsenved. Moreover, a marked increase in the clinical pregnancy rate per oocyte retrie$\mathrm{val}$ was noted. Now such treatment is used for $89 \%$ of all attempts, with a clinical pregnancy rate of $18.1 \%$ The increasing number of embryos was followed not only by the increase in the number of transferred embryos (2.2 for 1986 to 2.9 for 1989), but also an increase in frozen supernumerary embryos with a transfer at a later date, and weaker result (clinical pregnancy rate $7.5 \%$ per embryo transfer; French register). A major problem concems the high number of multiple pregnancies which accounted for about $23.5 \%$ all deliveries. The complications of such multiple pregnancies (prematurity, low birthweight, perinatal death) raise the need to define an increasingly rigorous strategy of embryo transfer in order to limit their frequency without decreasing the global efficiency of IVF.

in vitro fertilization / embryo transfer / man

\section{INTRODUCTION}

Parmi les méthodes de procréations médicalement assistées (PMA), la técondation in vitro a connu un développement important depuis une dizaine d'années. Ainsi, en France, on peut dénombrer environ 25000 enfants nés depuis les années 1970 à la suite d'inséminations avec sperme de donneur, soit actuellement près de 3000 enfants par an (Fédération des CECOS, 1990). L'enquête la plus récente, exhaustive à l'échelon national, recense un peu plus de 11000 enfants nés au 31 décembre 1989 à la suite d'une FIVETE (fécondation in vitro et transfert d'embryon) ou d'une méthode qui en dérive, soit un peu plus de 4000 enfants pour l'année 1989 (Buvat et al, 1990). Chaque année en France naissent 800000 enfants, par conséquent les naissances obtenues en PMA représentent moins de $1 \%$ du total. Nous allons nous attacher à décrire les caractères généraux, les évolutions particulières sur le plan méthodologique et les résultats concernant les grossesses obtenues et ceux concernant les enfants nés.

\section{LE DÉVELOPPEMENT DE LA FIVETE CHEZ L'HOMME}

On dispose depuis plusieurs années de moyens d'évaluation fournis par les registres nationaux. C'est la cas pour l'Australie et la Nouvelle Zélande depuis 1984, pour le Royaume-Uni depuis 1987, pour les États-Unis depuis 1988. En France 2 types d'informations sont disponibles: les unes sont des paramètres globaux sur l'activité et les naissances obtenues en FIV par plus de $90 \%$ des centres réalisant des PMA (Buvat et al, 1990) dans notre pays (Groupe d'étude de la fécondation in vitro en France = GEFF, depuis 1986); les autres sont les données individuelles détaillées fournies pour chaque tentative (FIVNAT, 1990) par $65 \%$ des centres (FIVNAT, depuis 1986).

Quelques chiffres permettent d'évaluer ce développement:

- le registre australo-néozélandais (National Perinatal Statistics Unit, 1988) faisait état en 1987 d'une vingtaine de centres (1 Centre $/ 10^{6}$ habitants),

- le registre américain (Medical Research International, 1991) faisait état en 1990 de 183 centres ( 1 Centre / 1,3 $10^{6}$ habitants), 
- le registre britannique (MRC, 1990; Beral et al, 1990) faisait état de 31 centres en 1987 ( 1 Centre / 1,7 106 habitants),

- le registre français (GEFF) faisait état (Buvat et al, 1990) en 1989 de 115 centres parmi lesquels 63 centres agréés par les pouvoirs publics représentaient $86 \%$ de l'activité totale (1 Centre / 0,9 $10^{6}$ habitants). Le nombre de tentatives a plus que doublé en 4 ans (1986: 11000 vs 1989 : 24000 tentatives).

\section{CARACTÈRES GÉNÉRAUX: ÂGE, DURÉE D'INFÉCONDITÉ, ANTÉCÉDENTS OBSTÉTRICAUX}

\section{Age}

Les couples engagés dans ces PMA sont plus âgés que ceux qui conçoivent naturellement comme en témoignent aussi bien l'âge moyen $(33,9$ vs 28,8 ans pour la femme et 36,1 ans pour l'homme dans le registre anglais) (Beral et al, 1990), 33,3 ans pour la femme dans le registre français (FIVNAT, 1990)) que la répartition par classes d'âge (en particulier le fait que $35 \%$ des femmes pour le registre américain, $23 \%$ des femmes pour le registre australien, $30 \%$ des femmes pour le registre français et $40 \%$ des femmes pour le registre britannique appartenaient à la classe d'âge $35 / 39$ ans).

\section{Durée d'infécondité}

C'est un autre paramètre important pour situer les techniques de PMA. La durée moyenne d'infécondité est de 6,3 ans pour le registre britannique, dans notre propre série elle atteint 7,4 ans.

\section{Antécédents obstétricaux}

Le registre britannique mentionne en particulier les antécédents de fausse-couche spontanée $(10 \%)$ et de grossesse extrautérine $(13 \%)$.

Au total, ces caractères généraux laissent présager des facteurs de risque obstétricaux, qu'il faut garder à l'esprit pour l'évolution des grossesses obtenues.

\section{ÉVOLUTION DES INDICATIONS}

Avant d'évoquer les indications proprement dites, il convient de préciser la place occupée par les méthodes dérivées de la fécondation in vitro (Gamete IntraFallopian Transfer, Zygote Intra-Fallopian Transfer, Tubal Embryo Transfer). Le GIFT qui couvre de loin l'effectif le plus important, représente de $25 \%$ (NPSU, 1988) à moins de $5 \%$ (Buvat et al, 1990) des PMA (IA avec donneur non comprise).

L'évolution générale des indications est représentée dans le tableau I. L'élément le plus caractéristique est la diminution de la fréquence des indications tubaires puisqu'en France par exemple, elles représentaient près des $3 / 4$ en 1986, alors que maintenant elles ne représentent qu'un peu moins des $2 / 3$ de l'ensemble des indications. La comparaison avec les registres étrangers ne nous permet pas de noter cette évolution, mais on peut remarquer que la fréquence des indications tubaires est plus élevée pour les registres australonéozélandais et britannique qui couvrent les périodes les plus anciennes. Le "rattrapage" du cumul de ces indications sur les années qui ont précédé le développement de la FIV est une explication possible. Une autre explication tout aussi plausible est fournie par l'augmentation de certaines indications, en particulier les in- 
Tableau I. Comparaison des indications de FIV entre pays et évolution de leur fréquence respective avec le temps.

\begin{tabular}{|c|c|c|c|c|c|}
\hline $\begin{array}{l}\text { Cause de l'infécondité } \\
\text { réf }\end{array}$ & $\begin{array}{l}\text { FIVNAT } 1986 \\
\text { (FIVNAT } \\
\text { 1990) }\end{array}$ & $\begin{array}{l}\text { FIVNAT } 1985 \\
\begin{array}{l}\text { (FIVNAT } \\
\text { 1990) }\end{array}\end{array}$ & $\begin{array}{l}9 \text { Registre } \\
\text { USA 1989 } \\
\text { (MRI 1991) }\end{array}$ & $\begin{array}{c}\text { Registre } \\
\text { Australien 1979-1987 } \\
\text { (NPSU 1988) }\end{array}$ & $\begin{array}{c}\text { Registre } \\
\text { UK 1978-1987 } \\
\text { (Beral et al } \\
1990\end{array}$ \\
\hline $\begin{array}{l}\text { Trompes } \\
\text { obstruées } \\
\text { labsentes }\end{array}$ & $55,6 \%$ & $37,2 \%$ & $38,1 \%$ & $46,6 \%$ & $61 \%$ \\
\hline $\begin{array}{l}\text { Trompes altérées } \\
\text { + autres facteurs }\end{array}$ & $17,2 \%$ & $24,4 \%$ & & & \\
\hline $\begin{array}{l}\text { Indication masculine } \\
\text { isolée }\end{array}$ & $10,9 \%$ & $14,5 \%$ & $23,4 \%$ & $7,2 \%$ & $8 \%$ \\
\hline Endométriose & $2,8 \%$ & $2,2 \%$ & $24,4 \%$ & $6,4 \%$ & $6 \%$ \\
\hline Infécondité inexpliquée & $9,7 \%$ & $11,4 \%$ & $10,5 \%$ & $13,6 \%$ & $11 \%$ \\
\hline $\begin{array}{l}\text { FIV avec sperme } \\
\text { de donneur }\end{array}$ & $2,2 \%$ & $2,8 \%$ & ND & ND & ND \\
\hline Mixtes et autres & $1,3 \%$ & $7,3 \%$ & $3,5 \%$ & $26,4 \%$ & $8 \%$ \\
\hline
\end{tabular}

dications masculines et les infécondités multifactorielles (respectivement $+3,6 \%$ et $+6 \%$ en 4 ans pour le registre français FIVNAT). La fréquence plus élevée de l'endométriose dans le registre américain par rapport aux autres est probablement biaisée par le fait que l'endométriose a dans ce cas été considérée comme déterminante pour l'infécondité sans tenir compte des autres facteurs associés, alors que, par exemple, le registre français n'a placé là que les endométrioses isolées. Dans notre série personnelle (1 093 tentatives entre 1988 et 1990 ) cette fréquence passe de $0,4 \%$ à plus de $10 \%$ selon que l'on considère l'endométriose isolée ou associée à d'autres facteurs d'infécondité (résultats enregistrés dans FIVNAT). La même remarque peut s'appliquer pour les indications masculines apparemment plus représentées dans le registre américain que dans les autres.

\section{ÉVOLUTIONS MÉTHODOLOGIQUES}

Elles concernent principalement les conditions de prélèvement ovocytaire, les modalités du traitement de superovulation et la congélation embryonnaire.

\section{Le prélèvement ovocytaire}

II s'est beaucoup simplifié puisque l'on est passé en quelques années d'un recueil sous anésthésie générale et sous contrôle coelioscopique imposant une hospitalisation de $48 \mathrm{~h}$, à un recueil sous simple anal- 
gésie par voie vaginale avec échoguidage ne nécessitant plus qu'une hospitalisation de jour. En pratique cela a réduit la durée du prélèvement, diminué les risques encourus par la patiente et réduit le coût de la tentative (Hazout, 1990). Le registre français pour 1989 mentionnait $95 \%$ des prélèvements effectués par voie échographique (FIVNAT, 1990).

\section{Les traitements de superovulation}

Ils ont été progressivement dominés par l'utilisation combinée d'agonistes de la LHRH et d'hormones gonadotropes (hMG, hFSH d'origine urinaire). L'utilisation des agonistes a été liée au problème des pics spontanés prématurés de LH / FSH survenant au cours des stimulations par hormones gonadotropes associées ou non avec des antiestrogènes (10-20\% des cycles selon les séries). L'hypophysectomie médicale gonadotrope ainsi obtenue permettait de s'affranchir de la sécrétion endogène des hormones gonadotropes. Deux modalités ont été développées selon la durée du traitement par l'agoniste de la LHRH avant le début de la stimulation exogène. Le protocole long comporte un blocage de l'axe hypothalamo-hypophysoovarien sur 10-14 j (Neveu et al, 1987), le protocole court ne comporte qu'un décalage de $48 \mathrm{~h}$ entre traitement agoniste et stimulation exogène (Barrière et al, 1987).

L'évolution a été très rapide puisqu'en France par exemple les agonistes qui n'étaient associés à la stimulation que pour $6,0 \%$ des traitements en 1986 , représentaient en $1989,89 \%$ des traitements (FIVNAT, 1990). Le registre américain pour cette même année faisait état de $73 \%$ de traitements utilisant des agonistes (Medical Research International, 1991). Cette différence s'explique par le développement plus tardif de ce traitement aux USA.
Ce développement s'explique par les conséquences moins attendues de ce traitement, la principale étant l'augmentation du pourcentage de grossesse obtenue. En effet, non seulement la fréquence des cycles où la collecte n'est pas possible a diminué comme l'on pouvait s'y attendre mais on a observé une nette augmentation du nombre d'ovocytes recueillis (1986:3,8 $\pm 2,7$ vs $1989: 7,3 \pm 5,0$, FIVNAT (1989, 1990)), ainsi qu'une augmentation du nombre d'embryons obtenus (1986: 2,2 \pm 1,9 vs $1989: 3,4 \pm 3,5$, FIVNAT). Comme on peut le voir sur la figure 1, l'augmentation la plus nette du taux de grossesse clinique par ponction en France entre 1986 et $1987(12,7 \%$ à $16,3 \%)$ va de pair avec l'augmentation la plus nette de la fréquence d'utilisation des agonistes de la LHRH au cours des stimulations $(6,0 \%$ à $60,5 \%$ ). À titre comparatif, on peut remarquer que le taux de grossesse clinique par ponction dans le registre américain (Medical Research International) est passé de 16 à $18 \%$ entre 1988 et 1989 , tandis que dans le même intervalle la proportion d'utilisation des agonistes de la LHRH est passée de 41 à $73 \%$.

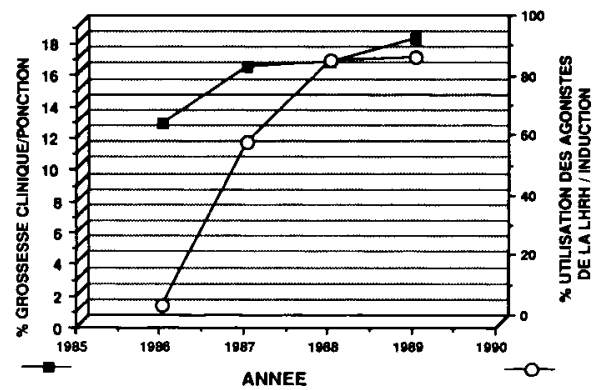

Fig 1. Évolution comparée de la fréquence d'utilisation des agonistes de la LHRH et des taux de grossesses cliniques en France (FIVNAT, 1990). 
Les raisons de cette augmentation ne sont pas claires, dans la mesure où l'augmentation du nombre d'embryons transférés qui est une conséquence logique de ce type de traitement (2,0 vs 2,9 entre 1986 et 1989) ne suffit pas à l'expliquer. En effet la comparaison entre protocole court et protocole long, sur les données nationales regroupant les années 1986 à 1989, révèle un avantage net du protocole long sur le court en terme de taux de grossesse clinique à nombre égal d'embryons transférés ( $27 \%$ vs $23,4 \%$ pour 3 embryons transférés, $p<0,001$; FIVNAT, 1990).

L'influence de ces traitements sur la qualité ovocytaire et la qualité embryonnaire a fait l'objet de controverses (Testart et al, 1989 ; Plachot et al, 1990). Quoiqu'il en soit, les résultats des congélations embryonnaires plaident en faveur d'une fragilité accrue des embryons obtenus par les traitements utilisant les agonistes de la LHRH comparativement avec les autres traitements quel que soit le nombre d'ovocytes recueillis (\% embryons implantés par embryon décongelé transféré $6,9 \%$ vs $10,9 \%$ selon l'utilisation ou non des agonistes lors de la tentative pour un recueil de moins de 6 ovocytes par exemple; Testart, 1990).L'intervention d'un facteur endométrial reste actuellement une hypothèse.

\section{La congélation embryonnaire}

Proposée avec succès depuis 1983 (Trounson et Mohr, 1983), la technique de congélation des embryons obtenus en FIV, en excédent du nombre des embryons à transférer, s'est largement inspirée des méthodes utilisées chez l'animal avec d'emblée des conditions relativement différentes tenant à l'obtention des embryons totalement in vitro ( $v$ s la congélation d'embryons obtenus in vivo), mais aussi au stade de développement généralement at- teint par l'embryon au moment de cette congélation ( 4 blastomères vs le stade de blastocyste). II faut donc garder ces notions à l'esprit quand on considère l'efficacité relativement faible des transferts d'embryons congelés chez l'homme (taux de grossesse clinique par transfert respectivement de $7,5 \%(n=2137)$ et de $11 \%(n=$ 2124 ) pour les registres français (Buvat et al, 1990) et américain (Medical Research International, 1991) pour l'année 1989).

\section{RÉSULTATS}

Nous nous limiterons à l'évaluation du pourcentage de grossesse, de la fréquence des grossesses multiples ainsi qu'aux caractères généraux de l'évolution de ces grossesses, avec les objectifs et perspectives que cela comporte.

\section{Les taux de grossesse, la fréquence des grossesses multiples}

Les résultats globaux sont représentés dans le tableau II, ils sont relativement proches d'un pays à l'autre si l'on tient compte de l'ancienneté du recueil. Nous ne rentrerons pas dans les détails des indications, mais signalons simplement que dans l'enquête française (FIVNAT, 1990), les résultats les plus faibles s'observent pour les indications masculines $(14,7 \%)$, les plus élevés pour les FIV avec sperme congelé de donneur $(24,2 \%)$ en terme de grossesse clinique par ponction.

L'autre résultat très important est constitué par la fréquence des grossesses multiples (tableau III), qui dépasse largement la fréquence des conceptions naturelles ( $1 \%$ dans les enquêtes britannique et française, Warnock Report, 1984; Rumeau et al, 1984). 
Tabieau II. Résultats globaux des tentatives de FIV à partir de 3 registres (NPSU, 1988; MRI, 1991; FIVNAT, 1990).

\begin{tabular}{lccc}
\hline $\begin{array}{l}\text { Registre année } \\
\text { (nb ponctions) }\end{array}$ & $\begin{array}{c}\text { Australie (1987) } \\
(\mathrm{n}=3921)\end{array}$ & $\begin{array}{c}\text { USA (1989) } \\
(\mathrm{n}=15392)\end{array}$ & $\begin{array}{c}\text { France 1986-1988 } \\
(\mathrm{n}=50976)\end{array}$ \\
\hline Grossesse clinique par ponction & $\begin{array}{r}13,2 \% \\
9,5 \%\end{array}$ & $\begin{array}{c}18 \% \\
14 \%\end{array}$ & $\begin{array}{c}16,9 \% \\
13,9 \%\end{array}$ \\
\hline
\end{tabular}

Tableau III. Fréquence des grossesses multiples en FIV (NPSU, 1988; MRI, 1991; FIVNAT, 1990; MRC, 1990; Beral et al, 1990).

\begin{tabular}{lcccc}
\hline & $\begin{array}{c}\text { Australie 1979-1987 } \\
(\mathrm{n}=2536)\end{array}$ & $\begin{array}{c}\text { France 1986-1988 } \\
(\mathrm{n}=2924)\end{array}$ & $\begin{array}{c}\text { UK 1978-1987 } \\
(\mathrm{n}=1267)\end{array}$ & $\begin{array}{c}\text { USA 1989 } \\
(\mathrm{n}=696)\end{array}$ \\
\hline Grossesse gem (\%) & $18,9 \%$ & $18,7 \%$ & $19 \%$ & $32,7 \%$ \\
Gros triple ou + (\%) & $3,8 \%$ & $3,6 \%$ & $4 \%$ & \\
\hline
\end{tabular}

Parmi les facteurs influençant la fréquence des grossesses multiples, l'âge de la femme et le nombre d'embryons transférés ont un rôle déterminant (tableau IV). C'est ainsi que la fréquence des grossesses multiples passe de $39 \%$ en dessous de 25 ans à $5 \%$ à partir de 40 ans dans le registre américain (Medical $R e$ search International, 1991). Pour le registre français (FIVNAT, 1990) cette fréquence passe de $23,6 \%$ avant 35 ans à $11,1 \%$ à partir de 40 ans (grossesses gémellaires).

La fréquence des grossesses multiples, en particulier triples, augmente avec le nombre d'embryons transférés $(p<0,001$; FIVNAT, 1990). Enfin, le taux de fécondation semble également influencer cette fréquence de grossesses multiples $(18,9 \%$ vs $24,9 \%$ respectivement en dessous et à partir de $75 \%$ d'ovocytes fécondés; FIVNAT, 1990).
Tableau IV. Variation de la fréquence des grossesses multiples en fonction du nombre d'embryons transférés (Beral et al, 1990; FIVNAT, 1990).

\begin{tabular}{|c|c|c|c|c|c|c|c|}
\hline $\begin{array}{l}N b \\
\text { embryons } \\
\text { transférés }\end{array}$ & 1 & 2 & 3 & 4 & 5 & 6 & 7 \\
\hline $\begin{array}{l}\text { UK } \\
\% \text { G2 } \\
(1989)\end{array}$ & 0 & 15 & 23 & 28 & 21 & 26 & 17 \\
\hline$\%$ G3 ou + & 0 & 0,5 & 5 & 4 & 6 & 6 & 25 \\
\hline $\begin{array}{l}\text { France } \\
\% \text { G2 } \\
(1986-1988) \\
\% \text { G3 ou }+\end{array}$ & $\int_{0}^{1,7}$ & $\begin{array}{r}12,9 \\
0,4\end{array}$ & $\begin{array}{l}18 \\
3,4\end{array}$ & 23,1 & & 23,7 & \\
\hline
\end{tabular}

\section{L'évolution des grossesses en FIV}

L'évolution globale est résumée dans le tableau V. S'il est difficile d'interpréter la fré- 
Tableau V. Évolution générale des grossesses en FIV selon les registres.

\begin{tabular}{lcccc}
\hline & $\begin{array}{c}\text { Australie } \\
1978-1987 \\
(\mathrm{n}=3247)\end{array}$ & $\begin{array}{c}\text { France } \\
1986-1988 \\
(\mathrm{n}=2985)\end{array}$ & $\begin{array}{c}\text { Royaume Uni } \\
1988\end{array}$ & $\begin{array}{c}\text { États-Unis } \\
1988 \\
\text { (n = 2 243) }\end{array}$ \\
\hline Fausses couches spontanées (\%) & 22,9 & 16,9 & 21 & \\
Grosses extra-utérines (\%) & 5,6 & 6,4 & 4,8 & 5 \\
Accouchements (\%) & 71,5 & 76,7 & 74,2 & 71 \\
Malformations (\%) & 2,2 & 2,8 & 2,2 & ND \\
Sex Ratio & 1,03 & 1,12 & 1,07 & ND \\
\hline
\end{tabular}

quence des fausses couches spontanées par rapport aux conceptions naturelles (15\%; Rumeau et al, 1984), en revanche son augmentation avec l'âge est confirmée par les registres (moins de $20 \%$ avant 25 ans vs $40 \%$ ou plus à partir de 40 ans). La fréquence des grossesses extra-utérines est nettement supérieure aux $1 \%$ rapportés dans les différents pays évoqués ici. Les indications tubaires, l'association d'une trompe perméable et d'une trompe obstruée ou absente sont un facteur déterminant de cette élévation (Mayaux et al, 1988; National Perinatal Statistics Unit, 1988). Dans la limite des effectifs disponibles, la fréquence des malformations ne semble pas significativement différente de celle observée à la suite de conceptions naturelles (2,6\% en France, Rumeau et al, 1984). La même remarque s'applique pour le sex ratio (1,09; Rumeau et al, 1984).

L'influence des grossesses multiples est résumée dans le tableau VI, pour ce qui concerne la prématurité (durée de la grossesse < 37semaines), les poids inférieurs à $2500 \mathrm{~g}$ et la mortalité périnatale. Ces résultats amènent deux remarques:

- la fréquence de prématurité, petit poids à la naissance et de mortalité périnatale (in utéro notamment) est plus élevée que dans la population générale même en ne considérant que les grossesses uniques. L'âge
Tableau VI. FIV et naissances : prématurité, faible poids et mortalité périnatale selon le nombre d'enfants.

\begin{tabular}{|c|c|c|c|}
\hline Nombre d'enfants & 1 & 2 & $\geq 3$ \\
\hline \multicolumn{4}{|l|}{ Prématurité (\%) } \\
\hline $\begin{array}{l}\text { France } \\
\text { (pop générale) }\end{array}$ & $\begin{array}{l}10,2 \\
(5)\end{array}$ & 37,8 & 79,4 \\
\hline $\begin{array}{l}\text { Royaume-Uni } \\
\text { (pop générale) }\end{array}$ & $\begin{array}{l}13 \\
(6)\end{array}$ & $\begin{array}{l}57 \\
(38)\end{array}$ & 95 \\
\hline \multicolumn{4}{|l|}{ Poids $<2500 \mathrm{~g} \mathrm{( \% )}$} \\
\hline $\begin{array}{l}\text { France } \\
\text { (pop générale) }\end{array}$ & $\begin{array}{l}15,7 \\
(3,7)\end{array}$ & 58 & 95,3 \\
\hline $\begin{array}{l}\text { Royaume-Uni } \\
\text { (pop générale) }\end{array}$ & $\begin{array}{l}12 \\
(7)\end{array}$ & 55 & 94 \\
\hline \multicolumn{4}{|l|}{ Mort périnatale (\%o) } \\
\hline France & 30,4 & & \\
\hline (pop générale) & 11,9 & 30,5 & 57,5 \\
\hline Royaume-Uni & $(6,2)$ & & \\
\hline (pop générale) & 11,7 & 39,7 & 79,3 \\
\hline (pop générale) & $\begin{array}{l}11,7 \\
(10,8)\end{array}$ & 39,7 & 79,3 \\
\hline
\end{tabular}

plus élevé, la primiparité, les antécédents obstétricaux que nous avons évoqués au début, sont des éléments à prendre en compte pour expliquer ces différences, cependant l'absence de population de référence, l'absence d'une totale exhaustivité 
des observations rapportées rendent difficile l'interprétation de ces résultats;

- par contre l'influence délétère des grossesses multiples sur ces paramètres de morbidité est incontestable quelle que soit la population étudiée.

\section{CONCLUSIONS ET PERSPECTIVES}

La fécondation in vitro chez l'homme a connu un développement qui ne doit pas faire oublier que les techniques de procréation médicalement assistèe n'interviennent que pour moins de $1 \%$ des enfants nés. Depuis les années 1980 , il existe une augmentation sensible de son efficacité jugée en terme d'enfant vivant. La population concernée par cette méthode de PMA est particulière de sorte que le recueil exhaustif des données constitue un moyen d'évaluation plus sûr que la simple comparaison avec la population générale. L'objectif majeur à court comme à long terme reste la diminution de la fréquence des grossesses multiples qui constituent la cause la plus importante de morbidité des grossesses en FIV. Le développement d'une stratégie de plus en plus rigoureuse de transfert d'embryons prenant en compte les facteurs de risques (âge, taux de fécondation notamment) est une solution à court terme. À moyen ou plus long terme une appréhension plus correcte du potentiel de développement embryonnaire, peut-être grâce à des procédés tels que la coculture, permettraient à la fois de réduire encore le risque de grossesse multiple et d'aborder la congélation des embryons à un stade plus tardif de développement et peut-être avec de meilleures conditions de développement ultérieur.

\section{REFERENCES}

Barriere P, Lopes P, Boiffard J (1987) GnRH analogs in ovulation induction for in vitro ferti- lization: benefit of a short administration regimen. J In Vitro Fertil Embryo Transfer, 4, 6465.

Beral V, Doyle P, Tan SL, Mason BA, Campbell $S$ (1990) Outcome of pregnancies resulting from assisted conception. $\mathrm{Br}$ Med Bull 46, 753-768

Buvat J, Buvat-Herbaut M, Dancoine M (1990) Bilan des procréations médicalement assistées en France en 1989 (GEFF). Contracept Fert Sex 18, 583-587

Fédération des CECOS (1990) L'insémination artificielle. Masson, Paris

FIVNAT (1990) Bilan FIVNAT 1989 et bilan général 1986-1989. Contracept Fertil Sex 18, $588-600$

FIVNAT (1989) Analyse des résultats 1988. Contracept Fertil Sex 17, 685-697

Hazout A (1990) Technique de recueil ovocytaire pour procréation médicalement assistée. In: Stérilités féminines et procréations médicalement assistées (Hédon $\mathrm{B}$, Frydman R, Plachot M, eds) Doin, Paris, 55-63

Mayaux MJ, Cohen J, Guichard-Moscato ML, Schwartz D (1988) Ectopic pregnancies after IVF. Ann NY Acad Sci USA 54, 1-6

Medical Research International (1991) Society for Assisted Reproductive Technology, The American Fertility Society, in vitro fertilization - embryo transfer (IVF-ET) in the United States: 1989 results from the IVF-ET Registry. Fertil Steril 55, 14-23

MRC (1990) Working party on children conceived by in vitro fertilization births in Great Britain resulting from assisted conception, 1978-1987. Br Med J 300, 1229-1233

National Perinatal Statistics Unit (1988) IVF and GIFT pregnancies, Australia and New Zealand, 1987. Sydney, Australia

Neveu S, Hedon B, Bringer J (1987) Ovarian stimulation by a combination of a gonadotropin releasing hormone agonist and gonadotropins for in vitro fertilization. Fertil Steril 47, 639-643

Plachot M, Mandelbaum J, Junca AM, Cohen J, Salat-Baroux J (1990) Qualité de l'ovocyte et de l'embryon et résultat de la FIV. Contracept Fert Sex 18, 636-638

Rumeau - Rouquette C, Du Mazaubrun C, Rabarison Y (1984) Naitre en France. INSERM ed, Paris 
Testart J (1990) Qualité des ovocytes et stimulation ovarienne. Contracept Fertil Sex 18, 639-643

Testart J, Belaisch-Allart J, Forman R, Gazengel A, Strub N, Frydman R (1989) Influence of different stimulation treatments on oocyte characteristics and in vitro fertilizing ability. Human Reprod 4, 192-197
Trounson A, Mohr L (1983) Human pregnancy following cryopreservation, thawing and transfer of an eight-cell embryo. Nature 305, 707.709

Warnock Report (1984) Committee of inquiry into human fertilization and embryology. Report. 1984, HMSO, London 